\title{
Convergence Analysis of Legendre Pseudospectral Scheme for Solving Nonlinear Systems of Volterra Integral Equations
}

\author{
Emran Tohidi, ${ }^{1}$ O. R. Navid Samadi, ${ }^{1}$ and S. Shateyi ${ }^{2}$ \\ ${ }^{1}$ Young Researchers and Elite Club, Mashhad Branch, Islamic Azad University, Mashhad, Iran \\ ${ }^{2}$ Institute for Groundwater Studies, Faculty of Natural and Agricultural Sciences, University of the Free State, \\ Bloemfontein 9300, South Africa \\ Correspondence should be addressed to S. Shateyi; stanford.shateyi@univen.ac.za
}

Received 27 December 2013; Revised 1 July 2014; Accepted 16 July 2014; Published 12 August 2014

Academic Editor: Hagen Neidhardt

Copyright (C) 2014 Emran Tohidi et al. This is an open access article distributed under the Creative Commons Attribution License, which permits unrestricted use, distribution, and reproduction in any medium, provided the original work is properly cited.

\begin{abstract}
We are concerned with the extension of a Legendre spectral method to the numerical solution of nonlinear systems of Volterra integral equations of the second kind. It is proved theoretically that the proposed method converges exponentially provided that the solution is sufficiently smooth. Also, three biological systems which are known as the systems of Lotka-Volterra equations are approximately solved by the presented method. Numerical results confirm the theoretical prediction of the exponential rate of convergence.
\end{abstract}

\section{Introduction}

Volterra-type integral equations (VIEs) are the mathematical model of many evolutionary problems with memory arising from biology, chemistry, physics, and engineering. For instance, in several heat transfer problems in physics, the equations are usually replaced by systems of Volterra integral equations (SVIEs). Since just few of these equations (i.e., VIEs and SVIEs) can be solved analytically, it is often necessary to apply appropriate numerical techniques.

Among numerical approaches, spectral methods are very powerful tools for approximating the solutions of many kinds of differential equations arising in various fields of science and engineering [1-5]. Spectral (exponential) accuracy and ease of applying these methods are two effective properties which have encouraged many authors to use them for integral equations (IEs) too. Spectral methods have been widely used by many authors in numerical analysis [6-13] for different kinds of IEs. In [11], Tang et al. proposed a Legendre spectral method (LSM) and its error analysis for the linear VIEs of the second kind. In this paper, we extend the LSM [11] to the numerical solution of the SVIEs of the second kind, including giving a convergence analysis for the nonlinear case. Thus, we consider the following nonlinear SVIEs:

$$
U(x)=\int_{-1}^{x} K(x, s, U(s)) d s+g(x), \quad-1 \leq x \leq 1,
$$

where $K(x, s, U(s))=\left[K_{1}(x, s, U(s)), K_{2}(x, s, U(s))\right]^{T}$ and $g(x)=\left[g_{1}(x), g_{2}(x)\right]^{T}$ are given, whereas $U(x)=[u(x)$, $v(x)]^{T}$ is the unknown function. We will consider the case that the solution of (1) is sufficiently smooth.

The remainder of this paper is organized as follows. The LSM is introduced in Section 2. Convergence analysis of the proposed method is discussed in Section 3. Section 4 states three applications of the desired equation in the biological systems. In Section 5, four types of biological models that are known as Lotka-Volterra system of equations are solved by the LSM to show the efficiency of the presented method and to verify the theoretical results obtained in Section 3. Also some comparisons are made with the existing results. Finally, Section 6 includes some concluding remarks. 


\section{Implementation of the Legendre Spectral Method}

In this section, we apply the basic idea of Tang et al. [11], which was previously used by the authors in $[10,12]$, for discretizing the nonlinear SVIEs (1). In the procedure of approximation, Legendre Gauss quadrature rule together with Lagrange interpolation is used.

In order to use the spectral method, we consider the collocation points $\left\{x_{i}\right\}_{i=0}^{N}$ as the set of $(N+1)$ Legendre-Gauss points (i.e., the roots of $L_{N+1}(x)=0$, where $L_{N+1}(x)$ is the $(N+1)$ th Legendre polynomial). Assume that the system (1) holds at $x_{i}$ :

$$
\begin{array}{r}
u\left(x_{i}\right)=\int_{-1}^{x_{i}} K_{1}\left(x_{i}, s, u(s), v(s)\right) d s+g_{1}\left(x_{i}\right), \\
v\left(x_{i}\right)=\int_{-1}^{x_{i}} K_{2}\left(x_{i}, s, u(s), v(s)\right) d s+g_{2}\left(x_{i}\right), \\
0 \leq i \leq N .
\end{array}
$$

Gauss quadrature rules can be used to compute approximately the integral terms in (2). To this end, we make the change of variable

$$
s=s\left(x_{i}, \theta\right)=\frac{x_{i}+1}{2} \theta+\frac{x_{i}-1}{2}, \quad-1 \leq \theta \leq 1,
$$

and obtain

$$
\begin{array}{r}
u\left(x_{i}\right)=\frac{x_{i}+1}{2} \int_{-1}^{1} K_{1}\left(x_{i}, s\left(x_{i}, \theta\right), u\left(s\left(x_{i}, \theta\right)\right),\right. \\
\left.v\left(s\left(x_{i}, \theta\right)\right)\right) d \theta+g_{1}\left(x_{i}\right), \\
v\left(x_{i}\right)=\frac{x_{i}+1}{2} \int_{-1}^{1} K_{2}\left(x_{i}, s\left(x_{i}, \theta\right), u\left(s\left(x_{i}, \theta\right)\right),\right. \\
\left.v\left(s\left(x_{i}, \theta\right)\right)\right) d \theta+g_{2}\left(x_{i}\right), \\
0 \leq i \leq N .
\end{array}
$$

By applying the $(N+1)$-point Gauss quadrature formula associated with the Legendre weights $\left\{w_{j}\right\}$, we get

$$
\begin{array}{r}
u\left(x_{i}\right) \approx \frac{x_{i}+1}{2} \sum_{j=0}^{N} K_{1}\left(x_{i}, s_{i}^{j}, u\left(s_{i}^{j}\right), v\left(s_{i}^{j}\right)\right) w_{j}+g_{1}\left(x_{i}\right), \\
v\left(x_{i}\right) \approx \frac{x_{i}+1}{2} \sum_{j=0}^{N} K_{2}\left(x_{i}, s_{i}^{j}, u\left(s_{i}^{j}\right), v\left(s_{i}^{j}\right)\right) w_{j}+g_{2}\left(x_{i}\right), \\
0 \leq i \leq N,
\end{array}
$$

where $s_{i}^{j}=s\left(x_{i}, \theta_{j}\right)$ and the points $\left\{\theta_{j}\right\}_{j=0}^{N}$ coincide with the collocation points $\left\{x_{j}\right\}_{j=0}^{N}$.

$$
\begin{aligned}
& \text { Let } u\left(x_{i}\right)=u_{i} \text { and } v\left(x_{i}\right)=v_{i}, \text { for } i=0,1, \ldots, N, \text { and } \\
& \qquad \begin{array}{c}
u(\sigma) \approx u_{N}(\sigma):=\sum_{k=0}^{N} u_{k} F_{k}(\sigma), \\
v(\sigma) \approx v_{N}(\sigma):=\sum_{k=0}^{N} v_{k} F_{k}(\sigma)
\end{array}
\end{aligned}
$$

represent the Lagrange interpolation polynomials of $u$ and $v$, in which $F_{k}$ is the $k$ th Lagrange basis function. The use of these interpolation polynomials for representing $u\left(s_{i}^{j}\right)$ and $v\left(s_{i}^{j}\right)$ in terms of $u_{i}$ and $v_{i}$ implies that

$$
\begin{aligned}
u_{i} \approx & \frac{x_{i}+1}{2} \sum_{j=0}^{N} K_{1}\left(x_{i}, s_{i}^{j}, \sum_{k=0}^{N} u_{k} F_{k}\left(s_{i}^{j}\right), \sum_{k=0}^{N} v_{k} F_{k}\left(s_{i}^{j}\right)\right) w_{j} \\
& +g_{1}\left(x_{i}\right), \\
v_{i} \approx & \frac{x_{i}+1}{2} \sum_{j=0}^{N} K_{2}\left(x_{i}, s_{i}^{j}, \sum_{k=0}^{N} u_{k} F_{k}\left(s_{i}^{j}\right), \sum_{k=0}^{N} v_{k} F_{k}\left(s_{i}^{j}\right)\right) w_{j} \\
& +g_{2}\left(x_{i}\right),
\end{aligned}
$$

$$
0 \leq i \leq N \text {. }
$$

Let $U_{i}$ and $V_{i}$ denote the approximation of $u_{i}$ and $v_{i}$, respectively. Then, from (7), we obtain the discrete problem

$$
\begin{aligned}
U_{i}= & \frac{x_{i}+1}{2} \sum_{j=0}^{N} K_{1}\left(x_{i}, s_{i}^{j}, \sum_{k=0}^{N} U_{k} F_{k}\left(s_{i}^{j}\right), \sum_{k=0}^{N} V_{k} F_{k}\left(s_{i}^{j}\right)\right) w_{j} \\
& +g_{1}\left(x_{i}\right), \\
V_{i}= & \frac{x_{i}+1}{2} \sum_{j=0}^{N} K_{2}\left(x_{i}, s_{i}^{j}, \sum_{k=0}^{N} U_{k} F_{k}\left(s_{i}^{j}\right), \sum_{k=0}^{N} V_{k} F_{k}\left(s_{i}^{j}\right)\right) w_{j} \\
& +g_{2}\left(x_{i}\right), \\
& 0 \leq i \leq N,
\end{aligned}
$$

which is a system of $2(N+1)$ nonlinear algebraic equations and $2(N+1)$ unknown coefficients $\left\{U_{i}\right\}_{i=0}^{N}$ and $\left\{V_{i}\right\}_{i=0}^{N}$. The nonlinear system (8) can be solved by an appropriate numerical method and the Lagrange interpolation of the solutions can be then obtained from (6). The numerical experiments show that the nonlinear system (8) can be solved easily by fsolve command in the Maple software.

\section{Convergence Analysis}

In this section, convergence analysis of the proposed method for the system of Volterra integral equations (1) will be provided. We show that the rate of convergence is exponential.

For convenience, we need some definitions and lemmas for providing the proof of the main Theorem. These lemmas 
include integral error of the Gauss quadrature rules, estimates of the interpolation error, Lebesgue constant of the Legendre series, and finally the Gronwall inequality.

Definition 1 . Let $I$ be a bounded interval of $\mathfrak{R}$, and let $1 \leq$ $p<+\infty$. One denotes by $L^{p}(I)$ the space of the measurable functions $u: I \rightarrow \Re$ such that $\int_{a}^{b}|u(x)|^{p} d x<+\infty$. Endowed with the norm

$$
\|u\|_{L^{p}(I)}=\left(\int_{a}^{b}|u(x)|^{p} d x\right)^{1 / p},
$$

it is a Banach space.

Definition 2. Let $I$ be a bounded interval of $\Re$, and let $m \geq 0$ be an integer. One defines $H^{m}(I)$ to be the vector space of the functions $v \in L^{2}(I)$ such that all the distributional derivatives of $u$ of order up to $m$ can be represented by functions in $L^{2}(I)$. In short,

$$
H^{m}(I)=\left\{u \in L^{2}(I): \frac{d^{k} u}{d x^{k}} \in L^{2}(I) \text {, for } 0 \leq k \leq m\right\} .
$$

$H^{m}(I)$ is endowed with the inner product

$$
(u, v)_{m}=\sum_{k=0}^{m} \int_{a}^{b} \frac{d^{k} u}{d x^{k}}(x) \frac{d^{k} v}{d x^{k}}(x) d x
$$

for which $H^{m}(I)$ is a Hilbert space. The associated norm is

$$
\|v\|_{H^{m}(I)}=\left(\sum_{k=0}^{m}\left\|\frac{d^{k} v}{d x^{k}}\right\|_{L^{2}(I)}\right)^{1 / 2} .
$$

Lemma 3 (integration error from Gauss quadrature [14, page 290]). Assume that a $(N+1)$-point Gauss or Gauss-Radau or Gauss-Lobatto quadrature formula relative to the Legendre weight is used to integrate the product $u \phi$, where $u \in H^{m}(I)$ with $I:=(-1,1)$ for some $m \geq 1$ and $\phi \in \mathscr{P}_{N}$. Then, there exists a constant $C$ independent of $N$ such that

$$
\left|\int_{-1}^{1} u(x) \phi(x) d x-(u, \phi)_{N}\right| \leq C N^{-m}|u|_{\widetilde{H}_{m, N}(I)}\|\phi\|_{L^{2}(I)},
$$

where

$$
\begin{gathered}
|u|_{\widetilde{H}_{m, N}(I)}=\left(\sum_{j=\min (m, N+1)}^{m}\left\|u^{(j)}\right\|_{L^{2}(I)}^{2}\right)^{1 / 2}, \\
(u, \phi)_{N}=\sum_{j=0}^{N} \omega_{j} u\left(x_{j}\right) \phi\left(x_{j}\right) .
\end{gathered}
$$

Lemma 4 (estimates for the interpolation error [14, page 289]). Assume that $u \in H^{m}(I)$ and denote $I_{N}(u)$ as its interpolation polynomial associated with the $(N+1)$-point Gauss or Gauss-Radau or Gauss-Lobatto points $\left\{x_{j}\right\}_{j=0}^{N}$, namely,

$$
I_{N}(u)=\sum_{i=0}^{N} u\left(x_{i}\right) F_{i}(x)
$$

Then, the following estimates hold:

$$
\begin{gathered}
\left\|u-I_{N}(u)\right\|_{L^{2}(I)} \leq C N^{-m}|u|_{\widetilde{H}_{m, N}(I)}, \\
\left\|u-I_{N}(u)\right\|_{H^{l}(I)} \leq C N^{2 l-1 / 2-m}|u|_{\widetilde{H}_{m, N}(I)}, \quad 1 \leq l \leq m .
\end{gathered}
$$

Lemma 5 (Lebesgue constant for the Legendre series [15]). Assume that $F_{j}(x)$ is the Nth Lagrange interpolation polynomials associated with the Gauss or Gauss-Radau or GaussLobatto points. Then,

$$
\max _{x \in(-1,1)} \sum_{j=0}^{N}\left|F_{j}(x)\right|=1+\frac{2^{3 / 2}}{\sqrt{\pi}} N^{1 / 2}+B_{0}+\mathcal{O}\left(N^{-1 / 2}\right),
$$

where $B_{0}$ is a bounded constant.

Lemma 6 (Gronwall inequality). If a nonnegative integrable function $E(t)$ satisfies

$$
E(t) \leq C_{1} \int_{-1}^{t} E(s) d s+G(t), \quad-1<t \leq 1,
$$

where $G(t)$ is an integrable function, then

$$
\|E\|_{L^{p}(I)} \leq C\|G\|_{L^{p}(I)}, \quad p \geq 1 .
$$

Here, we assume that the kernel $K(x, s, U(s))$ has the two following properties which are required for the proof of the convergence analysis:

(i) the Lipschitz property; in other words

$$
\begin{array}{r}
|K(x, s, \widehat{U}(s))-K(x, s, U(s))| \leq L_{K}|\widehat{U}(s)-U(s)|, \\
\forall \widehat{U}, U \in C[-1,1],
\end{array}
$$

where $U(s)=[u(s), v(s)]^{T}$ and $\widehat{U}(s)=[\widehat{u}(s), \widehat{v}(s)]^{T} ;$

(ii) $K(x, s, 0)=0_{2 \times 1}$.

In the following, we will provide the main theorem of this section in $L^{2}$. A similar technique could be designed in $L^{\infty}$ by using an extrapolation between $L^{2}$ and $H^{1}$ [11].

Theorem 7. Let $U$ be the exact solution of Volterra equation (1) and assume that

$$
U^{N}(x)=\sum_{j=0}^{N} \widehat{U}_{j} F_{j}(x),
$$

where $\widehat{U}_{j}=\left[U_{j}, V_{j}\right]^{T}(j=0,1, \ldots, N)$ is given by (8) and $F_{j}(x)$ is the jth Lagrange basis function associated with the Gauss points $\left\{x_{j}\right\}_{j=0}^{N}$. If $U \in H^{m}(I)$, then, for $m \geq 1$, we have

$$
\begin{aligned}
\| U- & U^{N} \|_{L^{2}(I)} \\
\leq & C N^{-m+1 / 2} \max _{x \in I}|K(x, s(x, \cdot), U(s(x, \cdot)))|_{\widetilde{H}_{m, N}(I)} \\
& +C N^{-m}|U|_{\widetilde{H}_{m, N}(I)},
\end{aligned}
$$

provided that $N$ is sufficiently large, where $C$ is a constant independent of $N$. 
Proof. According to notation (15), if

$$
\begin{aligned}
& \left(K\left(x, s, U^{N}(s)\right)\right)_{N, s} \\
& :=\sum_{j=0}^{N} K\left(x, s\left(x, \theta_{j}\right), U^{N}\left(s\left(x, \theta_{j}\right)\right)\right) w_{j},
\end{aligned}
$$

then the numerical schemes (5) can be written as

$$
\widehat{U}_{i}-\frac{1+x_{i}}{2}\left(K\left(x_{i}, s, U^{N}(s)\right)\right)_{N, s}=g\left(x_{i}\right), \quad 0 \leq i \leq N,
$$

which gives

$$
\begin{gathered}
\widehat{U}_{i}-\frac{1+x_{i}}{2} \int_{-1}^{1} K\left(x_{i}, s\left(x_{i}, \theta\right), U^{N}\left(s\left(x_{i}, \theta\right)\right)\right) d \theta \\
=g\left(x_{i}\right)+J_{1}\left(x_{i}\right), \quad 0 \leq i \leq N,
\end{gathered}
$$

where

$$
\begin{aligned}
J_{1}(x)= & -\frac{1+x}{2} \int_{-1}^{1} K\left(x, s(x, \theta), U^{N}(s(x, \theta))\right) d \theta \\
& +\frac{1+x}{2}\left(K\left(x, s, U^{N}(s)\right)\right)_{N, s} .
\end{aligned}
$$

From Lemma 3 and the assumption $x \leq 1($ or $(1+x) / 2 \leq 1)$, we have

$$
\begin{aligned}
\left|J_{1}(x)\right| & \leq \frac{1+x}{2} C N^{-m}\left|K\left(x, s(x, \cdot), U^{N}(s(x, \cdot))\right)\right|_{\widetilde{H}_{m, N}(I)} \\
& \leq C N^{-m}\left|K\left(x, s(x, \cdot), U^{N}(s(x, \cdot))\right)\right|_{\widetilde{H}_{m, N}(I)} .
\end{aligned}
$$

On the other hand, (27) can be rewritten as follows:

$$
\widehat{U}_{i}-\int_{-1}^{x_{i}} K\left(x, s, U^{N}(s)\right) d s=g\left(x_{i}\right)+J_{1}\left(x_{i}\right), \quad 0 \leq i \leq N .
$$

Multiplying $F_{j}(x)$ on both sides of (30) and summing up from 0 to $N$ yield

$$
U^{N}(x)-I_{N}\left(\int_{-1}^{x} K\left(x, s, U^{N}(s)\right) d s\right)=I_{N}(g)+I_{N}\left(J_{1}\right),
$$

which can be restated in the following form:

$$
\begin{aligned}
U^{N}(x) & -I_{N}\left(\int_{-1}^{x} K(x, s, U(s)) d s\right) \\
& -I_{N}\left(\int_{-1}^{x}\left[K\left(x, s, U^{N}(s)\right)-K(x, s, U(s))\right] d s\right) \\
= & I_{N}(g)+I_{N}\left(J_{1}\right),
\end{aligned}
$$

where $U^{N}$ is defined by (23) and the interpolation operator $I_{N}$ is defined by (16). It follows from (32) and (1) that

$$
\begin{aligned}
U^{N} & (x)+I_{N}(g-U) \\
& -I_{N}\left(\int_{-1}^{x}\left[K\left(x, s, U^{N}(s)\right)-K(x, s, U(s))\right] d s\right) \\
= & I_{N}(g)+I_{N}\left(J_{1}\right) .
\end{aligned}
$$

Let $e(x)=U^{N}(x)-U(x), x \in[-1,1]$, denote the error function. Then, we have

$$
\begin{aligned}
e(x) & +\left(U-I_{N} U\right)(x) \\
& -I_{N}\left(\int_{-1}^{x}\left[K\left(x, s, U^{N}(s)\right)-K(x, s, U(s))\right] d s\right) \\
= & I_{N}\left(J_{1}\right) .
\end{aligned}
$$

Consequently,

$$
\begin{aligned}
e(x)= & \int_{-1}^{x}\left[K\left(x, s, U^{N}(s)\right)-K(x, s, U(s))\right] d s \\
& +I_{N}\left(J_{1}\right)+J_{2}(x)+J_{3}(x),
\end{aligned}
$$

where

$$
\begin{gathered}
J_{2}(x)=I_{N} U(x)-U(x) \\
J_{3}(x)=-\int_{-1}^{x}\left[K\left(x, s, U^{N}(s)\right)-K(x, s, U(s))\right] d s \\
+I_{N}\left(\int_{-1}^{x}\left[K\left(x, s, U^{N}(s)\right)-K(x, s, U(s))\right] d s\right) .
\end{gathered}
$$

According to the Lipschitz property of the kernel $K$, we have

$$
\begin{aligned}
|e(x)| \leq & \left|\int_{-1}^{x}\left[K\left(x, s, U^{N}(s)\right)-K(x, s, U(s))\right] d s\right| \\
& +\left|I_{N}\left(J_{1}\right)+J_{2}(x)+J_{3}(x)\right| \\
\leq & L_{K} \int_{-1}^{x} \overbrace{\left[U^{N}(s)-U(s)\right]}^{e(s)} d s \\
& +\left|I_{N}\left(J_{1}\right)+J_{2}(x)+J_{3}(x)\right| .
\end{aligned}
$$

The use of the Gronwall inequality with $p=2$ yields

$$
\|e\|_{L^{2}(I)} \leq C\left\{\left\|I_{N}\left(J_{1}\right)\right\|_{L^{2}(I)}+\left\|J_{2}\right\|_{L^{2}(I)}+\left\|J_{3}\right\|_{L^{2}(I)}\right\} .
$$


From (29) and Lemma 5, we have

$$
\begin{aligned}
& \left\|I_{N}\left(J_{1}\right)\right\|_{L^{2}(I)} \\
& \leq C N^{-m} \max _{x \in I}\left|K\left(x, s(x, \cdot), U^{N}(s(x, \cdot))\right)\right|_{\widetilde{H}_{m, N}(I)} \\
& \quad \times \max _{x \in I} \sum_{j=0}^{N}\left|F_{j}(x)\right| \\
& \leq C N^{-m+1 / 2} \max _{x \in I}\left|K\left(x, s(x, \cdot), U^{N}(s(x, \cdot))\right)\right|_{\widetilde{H}_{m, N}(I)} \\
& \leq C N^{-m+1 / 2}
\end{aligned}
$$

According to the Lipschitz property of $K$

$$
\times \overbrace{\left(\max _{x \in I}|K(x, s(x, \cdot), U(s(x, \cdot)))|_{\widetilde{H}_{m, N}(I)}+L_{K}\|e\|_{L^{2}(I)}\right)} .
$$

Using $L^{2}$-error bounds for the interpolation polynomials (i.e., Lemma 4) gives

$$
\left\|J_{2}\right\|_{L^{2}(I)}=\left\|I_{N} U-U\right\|_{L^{2}(I)} \leq C N^{-m}|U|_{\widetilde{H}_{m, N}(I)} .
$$

By letting $m=1$ in (17), we have

$$
\begin{aligned}
\left\|J_{3}\right\|_{L^{2}(I)} & \leq L_{K}\left|\int_{-1}^{x} e(s) d s-I_{N}\left(\int_{-1}^{x} e(s) d s\right)\right| \\
& \leq C L_{K} N^{-1}\|e\|_{L^{2}(I)} .
\end{aligned}
$$

The above estimates, together with (38), yield

$$
\begin{aligned}
& \|e\|_{L^{2}(I)} \\
& \leq C N^{-m+1 / 2}\left(\max _{x \in I}|K(x, s(x, \cdot), U(s(x, \cdot)))|_{\widetilde{H}_{m, N}(I)}\right. \\
& \left.\quad+L_{K}\|e\|_{L^{2}(I)}\right) \\
& +C N^{-m}|U|_{\widetilde{H}_{m, N}(I)}+C L_{K} N^{-1}\|e\|_{L^{2}(I)}
\end{aligned}
$$

which leads to (24) provided that $N$ is sufficiently large. This completes the proof.

\section{Applications}

The Lotka-Volterra equations model the dynamic behavior of an arbitrary number of competitors [16]. Though originally formulated to describe the time history of a biological system, these equations find their application in a number of engineering fields such as nonlinear control. The accurate solutions of the Lotka-Volterra equations may become a difficult task either if the equations are stiff (even with a small number of species) or when the number of species is large [17]. Therefore, it is necessary to apply the robust numerical techniques to achieve the best approximations. We refer the interested reader to [18-22] for more information on the biological models and the Lotka-Volterra equations.
First, we consider the prey-predator model: LotkaVolterra system as an interacting species model to be governed by $[23,24]$

$$
\begin{aligned}
& \frac{d P_{1}}{d t}=P_{1}\left(a-b P_{2}\right), \\
& \frac{d P_{2}}{d t}=P_{2}\left(c P_{1}-d\right), \\
& P_{1}(0)=\lambda_{1}, \\
& P_{2}(0)=\lambda_{2}, \\
& 0 \leq t \leq b,
\end{aligned}
$$

where $a, b, c, d, \lambda_{1}$, and $\lambda_{2}$ are appropriate constants. Here, $P_{1}=P_{1}(t)$ is the prey (e.g., rabbits) population and $P_{2}=P_{2}(t)$ is the predator (e.g., foxes) population at time $t$.

As the second system, we consider the simple 2-species Lotka-Volterra competition model with each species $K_{1}$ and $K_{2}$ having logistic growth in the absence of the other $[23,24]$ :

$$
\begin{array}{r}
\frac{d K_{1}}{d \tau}=r_{1} K_{1}\left[1-\frac{K_{1}}{M_{1}}-c_{12} \frac{K_{2}}{M_{1}}\right], \\
\frac{d K_{2}}{d \tau}=r_{2} K_{2}\left[1-\frac{K_{2}}{M_{2}}-c_{21} \frac{K_{1}}{M_{2}}\right], \\
K_{1}(0)=\gamma_{1}, \quad K_{2}(0)=\gamma_{2},
\end{array}
$$

where $r_{1}, r_{2}, M_{1}, M_{2}, c_{12}, c_{21}, \gamma_{1}$, and $\gamma_{2}$ are all positive constants and the $r$ 's are the linear birth rates and the M's are the carrying capacities. The $c_{12}$ and $c_{21}$ measure the competitive effect of $K_{2}$ on $K_{1}$ and $K_{1}$ on $K_{2}$, respectively, and they are generally not equal. If we nondimensionalize this system by writing

$$
\begin{gathered}
P_{1}=\frac{K_{1}}{M_{1}}, \quad P_{2}=\frac{K_{2}}{M_{2}}, \quad t=r_{1} \tau, \\
\rho=\frac{r_{2}}{r_{1}}, \quad a=c_{12} \frac{M_{2}}{M_{1}}, \quad b=c_{21} \frac{M_{1}}{M_{2}},
\end{gathered}
$$

the system (44) would be changed into the following system:

$$
\begin{aligned}
& \frac{d P_{1}}{d t}=P_{1}\left(1-P_{1}-a P_{2}\right), \\
& \frac{d P_{2}}{d t}=\rho P_{2}\left(1-P_{2}-b P_{1}\right), \\
& P_{1}(0)=\lambda_{1}, \\
& P_{2}(0)=\lambda_{2}, \\
& 0 \leq t \leq 1,
\end{aligned}
$$

where $\lambda_{1}=\gamma_{1} / M_{1}$ and $\lambda_{2}=\gamma_{2} / M_{2}$ are the new initial values. 
As the final system, we consider the following version of the Lotka-Volterra equations [23]:

$$
\begin{aligned}
& \frac{d P_{1}}{d t}=P_{1}\left(1-P_{1}-\alpha P_{2}-\beta P_{3}\right), \\
& \frac{d P_{2}}{d t}=P_{2}\left(1-\beta P_{1}-P_{2}-\alpha P_{3}\right), \\
& \frac{d P_{3}}{d t}=P_{3}\left(1-\alpha P_{1}-\beta P_{2}-P_{3}\right), \\
& P_{1}(0)=\lambda_{1}, \\
& P_{2}(0)=\lambda_{2}, \\
& P_{3}(0)=\lambda_{3}, \\
& 0 \leq t \leq 1,
\end{aligned}
$$

where $\alpha, \beta, \lambda_{1}, \lambda_{2}$, and $\lambda_{3}$ are constants.

These models can be easily transformed into their associated systems of Volterra integral equations. This idea (i.e., changing the IVPs into their associated Volterra integral forms) has been done by many authors like [25, 26]. This approach of transforming the IVPs into their Volterra form has many interesting advantages such as imposing the initial conditions to the new equations and ease of applying high order Gauss quadrature rules for getting highly accurate approximations.

For instance, the first model (43) can be transformed into a system of Volterra integral equations as follows:

$$
\begin{array}{r}
P_{1}(t)=\int_{0}^{t} P_{1}(\tau)\left(a-b P_{2}(\tau)\right) d \tau+\lambda_{1}, \\
P_{2}(t)=\int_{0}^{t} P_{2}(\tau)\left(c P_{1}(\tau)-d\right) d \tau+\lambda_{2}, \\
0 \leq t \leq b .
\end{array}
$$

For the ease of applying the spectral method, as [11], we make the change of variable $\tau=b((1+x) / 2)$ and observe the problem

$$
\begin{array}{r}
u(x)=\int_{-1}^{x} u(s)(\bar{a}-\bar{b} v(s)) d s+\lambda_{1}, \\
v(x)=\int_{-1}^{x} v(s)(\bar{c} u(s)-\bar{d}) d s+\lambda_{2}, \\
-1 \leq x \leq 1,
\end{array}
$$

where

$$
\begin{aligned}
& u(x)=P_{1}\left(\frac{b(1+x)}{2}\right), v(x)=P_{2}\left(\frac{b(1+x)}{2}\right), \\
& \bar{a}=\frac{a b}{2}, \quad \bar{b}=\frac{b^{2}}{2}, \quad \bar{c}=\frac{b c}{2}, \quad \bar{d}=\frac{b d}{2} .
\end{aligned}
$$

Similar procedures can be applied to restate the other models (46)-(47) as Volterra integral equations system in $[-1,1]$.

\section{Numerical Examples}

In this section, some numerical examples are considered to illustrate the efficiency and accuracy of the proposed method. In all examples, we consider $\left\{x_{i}\right\}_{i=0}^{N}$ as the Legendre-Gauss points with the corresponding weights

$$
w_{i}=\frac{2}{\left(1-x_{i}^{2}\right)\left[L_{N+1}^{\prime}\left(x_{i}\right)\right]^{2}}, \quad i=0,1, \ldots, N,
$$

where $L_{N+1}(x)$ is the $(N+1)$ th Legendre polynomial. Also, the nonlinear algebraic systems are solved directly by using fsolve command in Maple 13 software with the Digits environment variable assigned to be 30 . All calculations are run on a Pentium 4 PC with 2.70 GHz CPU and 4 GB RAM. In order to show the efficiency of the LSM, we compare our results with those of the other methods that were proposed recently in the literature like Bessel collocation method [24] (BCM), HPM [27], and VIM [28]. It should be noted that, in all tables, the absolute values of residuals are provided in the uniform nodes $t=0, t=0.2, t=0.4, t=0.6, t=0.8$, and $t=1$.

Example 1. Let us first consider the following problem for model (43):

$$
\begin{aligned}
& \frac{d P_{1}}{d t}=P_{1}\left(1-P_{2}\right), \\
& \frac{d P_{2}}{d t}=P_{2}\left(P_{1}-1\right), \\
& P_{1}(0)=1.3 \\
& P_{2}(0)=0.6 \\
& 0 \leq t \leq 1,
\end{aligned}
$$

where $a=b=c=d=1, \lambda_{1}=1.3$, and $\lambda_{2}=0.6$. We transfer this model to the system of Volterra integral equations as follows:

$$
\begin{aligned}
& u(x)=\frac{1}{2} \int_{-1}^{x} u(s)(1-v(s)) d s+1.3 \\
& v(x)=\frac{1}{2} \int_{-1}^{x} v(s)(u(s)-1) d s+0.6
\end{aligned}
$$

$$
-1 \leq x \leq 1,
$$

where $u(x)=P_{1}((x+1) / 2)$ and $v(x)=P_{2}((x+1) / 2)$.

For comparing the LSM and BCM [24], for $N=3,6,9$, the absolute values of residual functions for the approximate solutions obtained by the LSM and BCM are provided in Table 5. These comparisons are also depicted in Figure 1. Moreover, Figure 2 displays the residual functions $E_{i, N}(t)=$ $\bar{E}_{i, N}(2 t-1) \quad(i=1,2)$ which are obtained by our method for $N=15$. From Table 1 and Figure 1, we observe that the presented method is very effective and the obtained results are better than those of the BCM. In [24], one can see that the BCM is better than the ADM [29] and HPM [27]; thus, the current method is more effective than these methods too. From Figure 2, one can conclude that numerical solution with high accuracy is furnished by the presented method. 




(a)

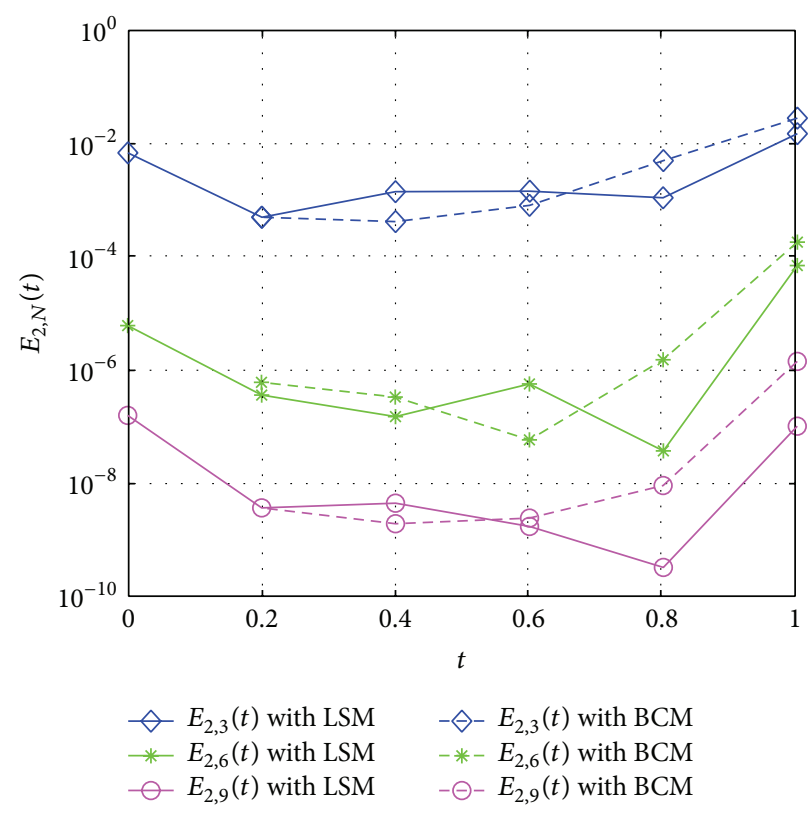

(b)

Figure 1: Comparison of the residuals at the selected points of Example 1, for $N=3,6$, and 9.

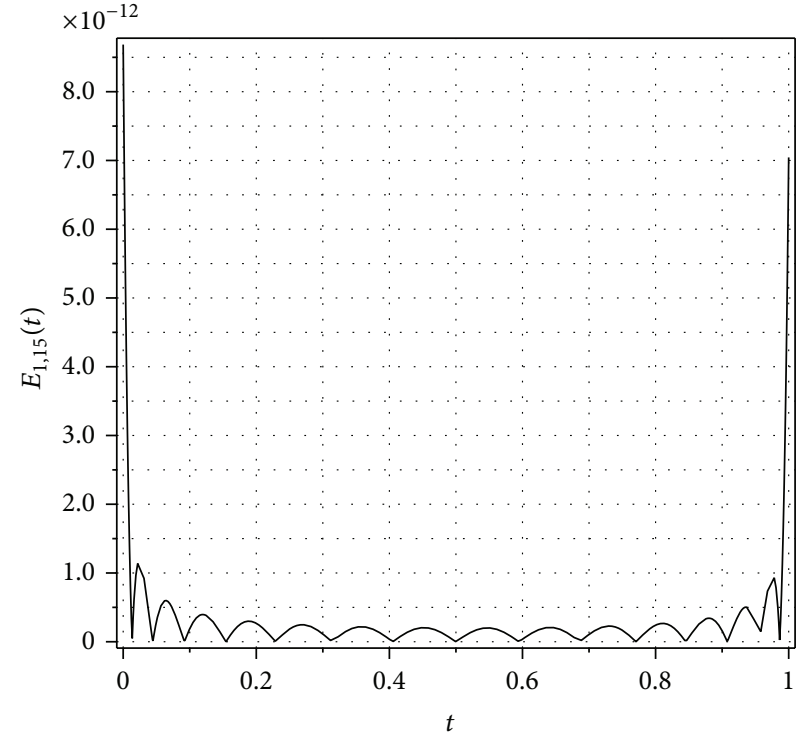

(a)



(b)

Figure 2: History of the residual functions of Example 1, for $N=15$, in the interval $[0,1]$.

Example 2. We now consider the following problem for model (46):

$$
\begin{aligned}
& P_{2}(0)=1, \\
& 0 \leq t \leq 1,
\end{aligned}
$$

$$
\begin{aligned}
& \frac{d P_{1}}{d t}=P_{1}\left(1-P_{1}-P_{2}\right), \\
& \frac{d P_{2}}{d t}=P_{2}\left(1-P_{2}-0.8 P_{1}\right), \\
& P_{1}(0)=1,
\end{aligned}
$$

where $a=\rho=\lambda_{1}=\lambda_{2}=1$ and $b=0.8$.

We apply the presented method to find the approximate solutions of the equivalent system of Volterra integral equations. Table 2 shows the numerical results of the residual 


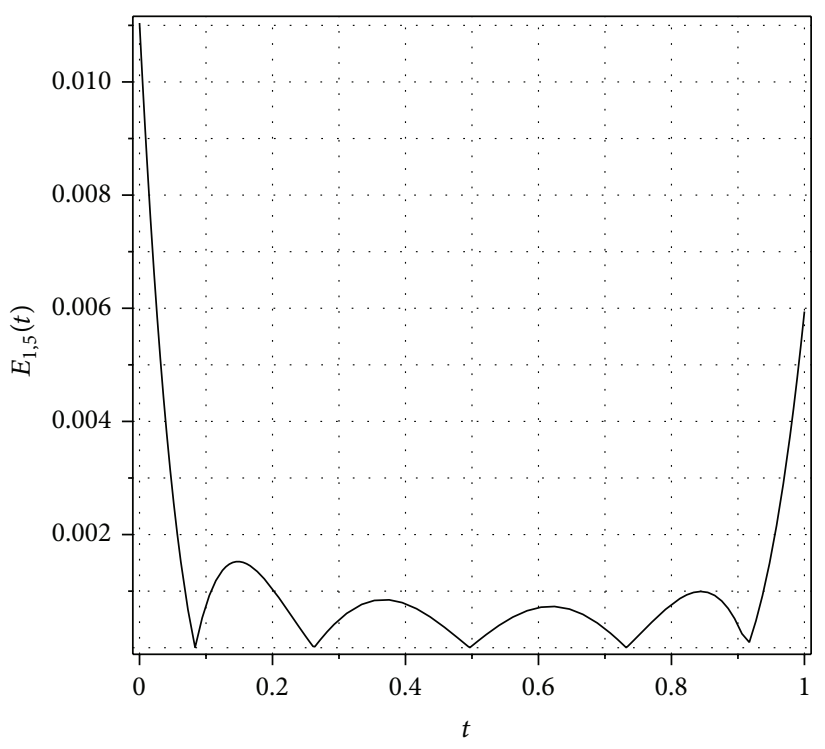

(a)



(b)

FIGURE 3: Residual functions $E_{1,5}(t)$ and $E_{2,5}(t)$ of the LSM in the interval $[0,1]$.

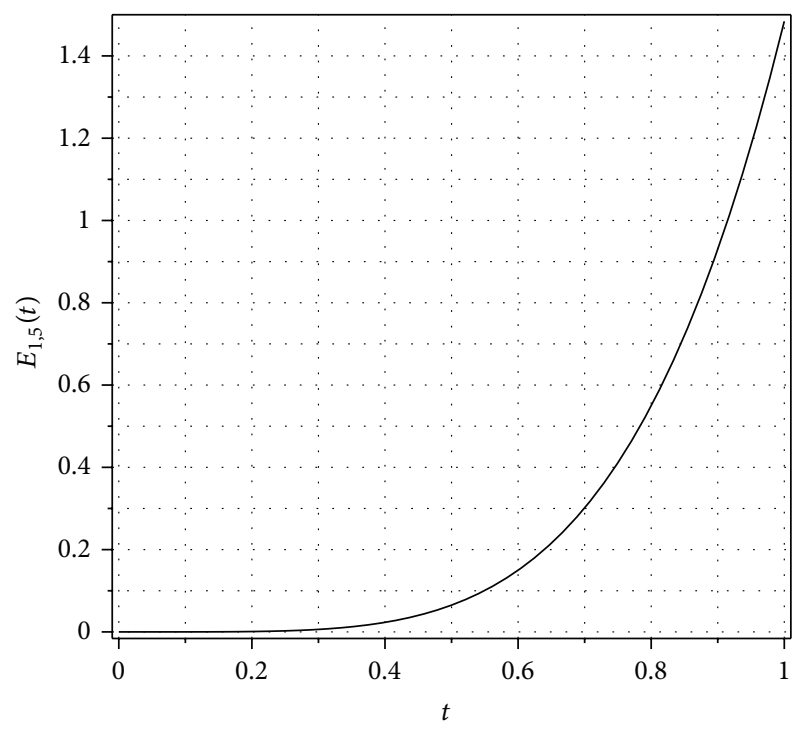

(a)

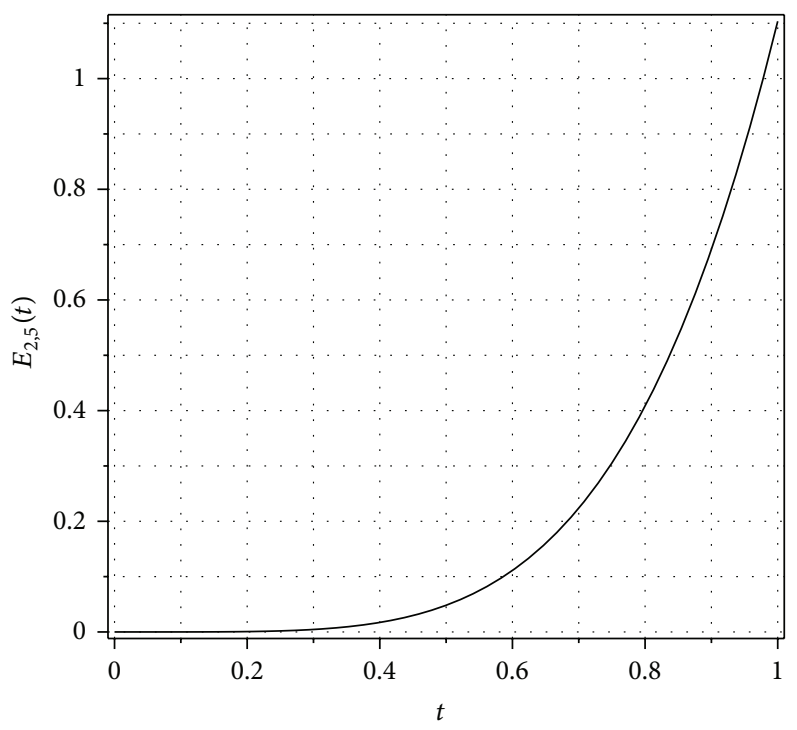

(b)

FIgURE 4: Residual functions $E_{1,5}(t)$ and $E_{2,5}(t)$ of the HPM in the interval $[0,1]$.

functions $E_{i, N}(t)(i=1,2)$ obtained by LSM for $N=5$, 10,15 . In order to compare the results of our method for $N=5$ with the five-term HPM solutions [27], the residual functions $E_{i, N}(t)(i=1,2)$ of these methods are depicted in Figures 3 and 4 . From Table 2, one can conclude that the LSM provides the numerical solutions with high accuracy. Also, from Figures 3 and 4, we see that the results obtained by the presented method are better than those obtained by the HPM.
Example 3. At this stage, we solve a typical system in model (47) using the LSM with $\alpha=\beta=0.1, \lambda_{1}=0.2, \lambda_{2}=0.3$, and $\lambda_{3}=0.5$ :

$$
\begin{aligned}
& \frac{d P_{1}}{d t}=P_{1}\left(1-P_{1}-0.1 P_{2}-0.1 P_{3}\right), \\
& \frac{d P_{2}}{d t}=P_{2}\left(1-0.1 P_{1}-P_{2}-0.1 P_{3}\right),
\end{aligned}
$$






(a)

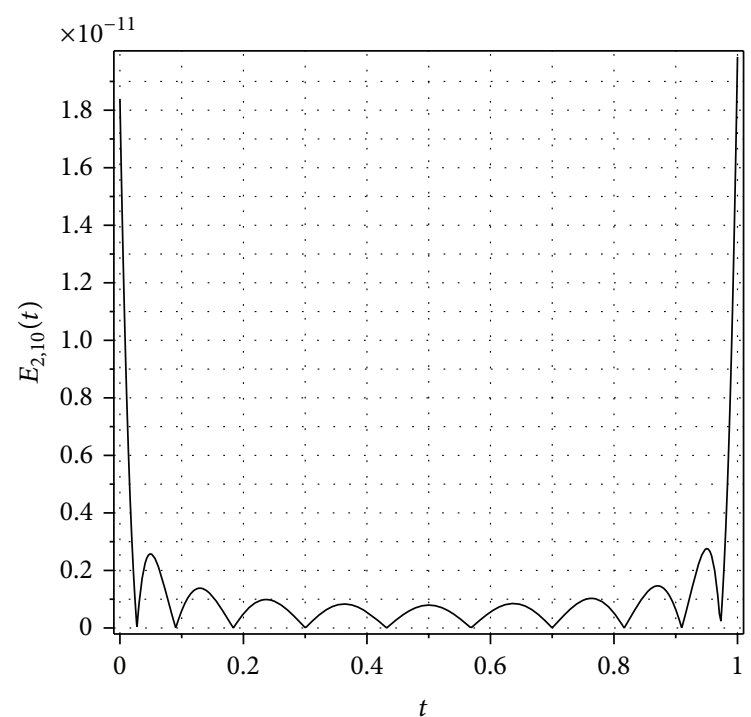

(b)

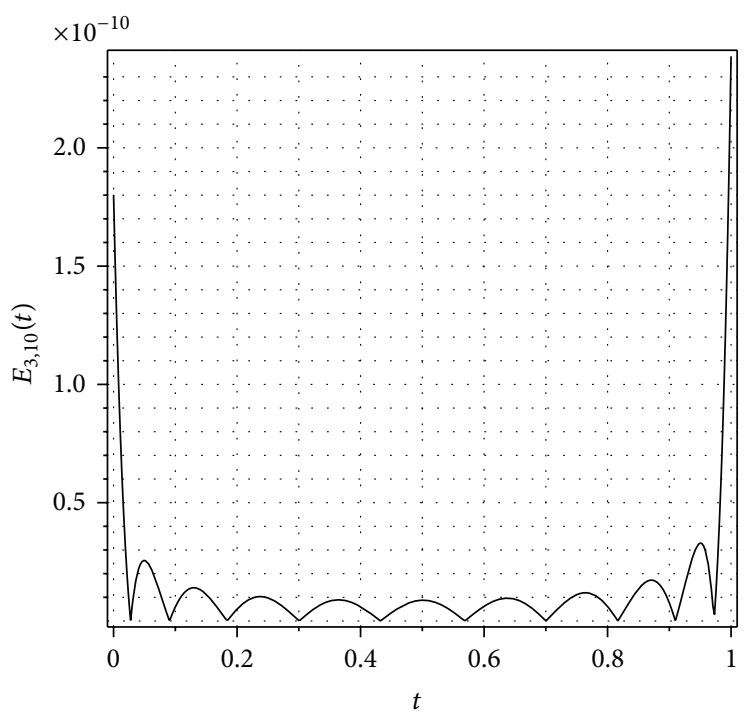

(c)

Figure 5: Residual functions $E_{1,10}(t), E_{2,10}(t)$, and $E_{3,10}(t)$ of LSM in the interval $[0,1]$.

$$
\begin{gathered}
\frac{d P_{3}}{d t}=P_{3}\left(1-0.1 P_{1}-0.1 P_{2}-P_{3}\right), \\
P_{1}(0)=0.2, \quad P_{2}(0)=0.3, \quad P_{3}(0)=0.5 .
\end{gathered}
$$

After transforming this system to the equivalent Volterra system, we apply the LSM for different values of $N$. Numerical values of the residual functions obtained by LSM are provided in Table 3. Moreover, for comparing with other methods, the numerical results of the variational iteration method (VIM) [28], the 4th-order Runge-Kutta method (RK4), and the LSM for $N=3$ are given in Table 4 . Also, Figure 5 displays the residual functions $E_{i, N}(x) \quad(i=1,2,3)$, for $N=10$ which are furnished by the LSM. From these tables and figures, we observe that the results of our method are very accurate and they are better than those obtained by the other methods.

Example 4. As the final example, we consider the following problem that is selected from [30]:

$$
\begin{aligned}
& \frac{d P_{1}(t)}{d t}=P_{1}(t)\left((4+\tan (t))-\exp (2 t) P_{2}(t)\right), \\
& \frac{d P_{2}(t)}{d t}=P_{2}(t)\left(2+\cos (t) P_{1}(t)\right), \\
& P_{1}(0)=-4, \\
& P_{2}(0)=4, \\
& 0 \leq t \leq 1,
\end{aligned}
$$


TABLE 1: Comparison of the residual functions of (53) for the $t_{i}$ values.

\begin{tabular}{|c|c|c|c|c|c|c|}
\hline$t_{i}$ & $E_{1,3}\left(t_{i}\right)$ & $E_{1,6}\left(t_{i}\right)$ & $E_{1,9}\left(t_{i}\right)$ & $E_{2,3}\left(t_{i}\right)$ & $E_{2,6}\left(t_{i}\right)$ & $E_{2,9}\left(t_{i}\right)$ \\
\hline \multicolumn{7}{|l|}{ LSM } \\
\hline 0 & $9.0 e-03$ & $7.4 e-05$ & $4.6 e-07$ & $7.0 e-03$ & $6.1 e-06$ & $1.9 e-07$ \\
\hline 0.2 & $6.7 e-04$ & $3.0 e-07$ & $1.2 e-08$ & $4.8 e-04$ & $3.7 e-07$ & $3.5 e-09$ \\
\hline 0.4 & $7.8 e-04$ & $6.1 e-07$ & $1.7 e-08$ & $1.4 e-03$ & $1.5 e-07$ & $4.4 e-09$ \\
\hline 0.6 & $7.4 e-04$ & $1.0 e-07$ & $1.4 e-08$ & $1.4 e-03$ & $5.6 e-07$ & $1.7 e-09$ \\
\hline 0.8 & $2.9 e-05$ & $7.1 e-07$ & $8.7 e-09$ & $1.1 e-03$ & $3.6 e-08$ & $3.2 e-10$ \\
\hline 1 & $5.4 e-04$ & $9.9 e-06$ & $1.9 e-07$ & $1.5 e-02$ & $7.0 e-05$ & $1.0 e-07$ \\
\hline \multicolumn{7}{|l|}{$t_{i}$} \\
\hline \multicolumn{7}{|l|}{$\mathrm{BCM}$} \\
\hline 0 & 0 & 0 & 0 & 0 & 0 & 0 \\
\hline 0.2 & $2.2 e-03$ & $3.2 e-06$ & $7.5 e-09$ & $5.4 e-04$ & $5.9 e-07$ & $3.7 e-09$ \\
\hline 0.4 & $1.2 e-03$ & $2.7 e-06$ & $4.0 e-09$ & $4.1 e-04$ & $3.3 e-07$ & $1.9 e-09$ \\
\hline 0.6 & $1.6 e-03$ & $3.6 e-06$ & $5.6 e-09$ & $8.1 e-04$ & $5.7 e-08$ & $2.4 e-09$ \\
\hline 0.8 & $6.1 e-03$ & $8.8 e-06$ & $2.4 e-08$ & $4.9 e-03$ & $1.5 e-06$ & $8.9 e-09$ \\
\hline 1 & $2.1 e-02$ & $3.3 e-04$ & $5.6 e-06$ & $2.8 e-02$ & $1.8 e-04$ & $1.4 e-06$ \\
\hline
\end{tabular}

TABLE 2: The residual functions $E_{i, N}(t)$ for the selected nodes of Example 2.

\begin{tabular}{|c|c|c|c|c|c|c|}
\hline$t_{i}$ & $E_{1,5}\left(t_{i}\right)$ & $E_{1,10}\left(x_{i}\right)$ & $E_{1,15}\left(t_{i}\right)$ & $E_{2,5}\left(t_{i}\right)$ & $E_{2,10}\left(t_{i}\right)$ & $E_{2,15}\left(t_{i}\right)$ \\
\hline 0 & $1.1 e-02$ & $2.0 e-05$ & $2.1 e-08$ & $8.2 e-03$ & $1.4 e-05$ & $1.4 e-08$ \\
\hline 0.2 & $1.0 e-03$ & $4.6 e-07$ & $5.1 e-10$ & $7.8 e-04$ & $3.2 e-07$ & $3.5 e-10$ \\
\hline 0.4 & $7.8 e-04$ & $3.9 e-07$ & $5.3 e-11$ & $5.9 e-04$ & $2.7 e-07$ & $3.6 e-11$ \\
\hline 0.6 & $7.1 e-04$ & $3.6 e-07$ & $5.9 e-11$ & $5.4 e-04$ & $2.6 e-07$ & $4.0 e-11$ \\
\hline 0.8 & $7.6 e-04$ & $2.8 e-07$ & $3.4 e-10$ & $5.9 e-04$ & $2.0 e-07$ & $2.3 e-10$ \\
\hline 1 & $5.9 e-03$ & $9.8 e-06$ & $9.7 e-09$ & $4.6 e-03$ & $7.1 e-06$ & $6.7 e-09$ \\
\hline
\end{tabular}

TABLE 3: The residual functions $E_{i, N}(t)$ of Example 3 for the selected nodes.

\begin{tabular}{|c|c|c|c|c|c|c|}
\hline$t_{i}$ & $E_{1,3}\left(t_{i}\right)$ & $E_{1,6}\left(t_{i}\right)$ & $E_{2,3}\left(t_{i}\right)$ & $E_{2,6}\left(t_{i}\right)$ & $E_{3,3}\left(t_{i}\right)$ & $E_{3,6}\left(t_{i}\right)$ \\
\hline 0 & $8.7 e-04$ & $1.4 e-07$ & $4.0 e-04$ & $2.1 e-06$ & $1.3 e-03$ & $4.2 e-07$ \\
\hline 0.2 & $6.3 e-05$ & $3.7 e-09$ & $3.0 e-05$ & $1.8 e-08$ & $9.5 e-05$ & $3.5 e-09$ \\
\hline 0.4 & $1.0 e-04$ & $2.1 e-10$ & $2.4 e-05$ & $1.1 e-08$ & $2.0 e-04$ & $4.9 e-09$ \\
\hline 0.6 & $1.0 e-04$ & $3.8 e-09$ & $2.2 e-05$ & $1.3 e-08$ & $2.0 e-04$ & $5.4 e-09$ \\
\hline 0.8 & $4.6 e-05$ & $1.5 e-09$ & $5.6 e-06$ & $1.8 e-08$ & $1.2 e-04$ & $2.1 e-09$ \\
\hline 1 & $6.5 e-04$ & $4.9 e-07$ & $6.3 e-05$ & $2.2 e-06$ & $1.6 e-03$ & $4.9 e-07$ \\
\hline
\end{tabular}

TABLE 4: Comparisons of the numerical solutions for Example 3.

\begin{tabular}{|c|c|c|c|c|c|c|c|c|c|}
\hline \multirow{2}{*}{$t_{i}$} & \multicolumn{3}{|c|}{ 4-iteration VIM } & \multicolumn{3}{|c|}{$\mathrm{RK} 4, h=0.001$} & \multicolumn{3}{|c|}{$\mathrm{LSM}, N=3$} \\
\hline & $u$ & $v$ & $z$ & $u$ & $v$ & $z$ & u & $v$ & $z$ \\
\hline 0 & 0.20000 & 0.30000 & 0.50000 & 0.20000 & 0.30000 & 0.50000 & 0.20004 & 0.30002 & 0.49993 \\
\hline 0.2 & 0.23010 & 0.33873 & 0.54429 & 0.23010 & 0.33873 & 0.54428 & 0.23008 & 0.33872 & 0.54431 \\
\hline 0.4 & 0.26265 & 0.37889 & 0.58662 & 0.26264 & 0.37888 & 0.58655 & 0.26265 & 0.37888 & 0.58653 \\
\hline 0.6 & 0.29734 & 0.41987 & 0.62679 & 0.29729 & 0.41974 & 0.62601 & 0.29730 & 0.41974 & 0.62599 \\
\hline 0.8 & 0.33393 & 0.46138 & 0.66683 & 0.33361 & 0.46054 & 0.66208 & 0.33359 & 0.46054 & 0.66211 \\
\hline 1 & 0.37256 & 0.50438 & 0.71455 & 0.37105 & 0.50053 & 0.69438 & 0.37108 & 0.50053 & 0.69430 \\
\hline
\end{tabular}


TABLE 5: Numerical results of Example 4.

\begin{tabular}{ccccc}
\hline$N$ & 10 & 11 & 12 & 13 \\
\hline$e_{N}$ & 0.018 & 0.0076 & 0.0025 & 0.00091 \\
\hline
\end{tabular}

with the exact solutions $P_{1}(t)=-4 / \cos (t)$ and $P_{2}(t)=$ $4 \exp (-2 t)$. Again, for solving this problem, we use several values of $N$ such as $10,11,12$, and 13 and obtain

$$
\begin{aligned}
e_{N}=\max \left\{\max _{0 \leq t \leq 1} \mid \frac{d P_{1}(t)}{d t}-P_{1}(t)\right. \\
\times\left((4+\tan (t))-\exp (2 t) P_{2}(t)\right) \mid, \\
\left.\max _{0 \leq t \leq 1}\left|\frac{d P_{2}(t)}{d t}-P_{2}(t)\left(2+\cos (t) P_{1}(t)\right)\right|\right\}
\end{aligned}
$$

for the mentioned values of $N$ and report them in Table 5.

\section{Conclusions}

This paper deals with the LSM for computing the approximate solution of the systems of nonlinear Volterra integral equations by using the Lagrange interpolations and Gauss quadrature rules. We demonstrated that the errors of the spectral approximations decay exponentially in the nonlinear case. The numerical results obtained for the solutions of the systems of the Lotka-Volterra equations confirm the spectral accuracy of the LSM. In addition, the comparisons of the residual functions obtained by our scheme with those obtained by other methods show that the LSM is more effective than the other methods.

\section{Conflict of Interests}

The authors declare that they do not have any conflict of interests in their submitted paper.

\section{References}

[1] A. Bhrawy, L. Assas, E. Tohidi, and M. Alghamdi, "A LegendreGauss collocation method for neutral functional-differential equations with proportional delays," Advances in Difference Equations, vol. 2013, article 63, 2013.

[2] J. P. Boyd, Chebyshev and Fourier Spectral Methods, Dover, Mineola, NY, USA, 2nd edition, 2001.

[3] C. Canuto, M. Y. Hussaini, A. Quarteroni, and T. A. Zang, Spectral Methods in Fluid Dynamics, Springer, New York, NY, USA, 1988.

[4] E. Tohidi, "Legendre approximation for solving linear HPDEs and comparison with Taylor and Bernoulli matrix methods," Applied Mathematics, vol. 3, no. 5, pp. 410-416, 2012.

[5] F. Toutounian, E. Tohidi, and A. Kilicman, "Fourier operational matrices of differentiation and transmission: introduction and applications," Abstract and Applied Analysis, vol. 2013, Article ID 198926, 11 pages, 2013.

[6] Y. Chen and T. Tang, "Spectral methods for weakly singular Volterra integral equations with smooth solutions," Journal of
Computational and Applied Mathematics, vol. 233, no. 4, pp. 938-950, 2009.

[7] G. N. Elnagar and M. Kazemi, "Chebyshev spectral solution of nonlinear Volterra-Hammerstein integral equations," Journal of Computational and Applied Mathematics, vol. 76, no. 1-2, pp. 147-158, 1996.

[8] Y. J. Jiang, "On spectral methods for Volterra-type integrodifferential equations," Journal of Computational and Applied Mathematics, vol. 230, no. 2, pp. 333-340, 2009.

[9] J. S. Nadjafi, O. R. N. Samadi, and E. Tohidi, "Numerical solution of two dimensional integral equations via a spectral Galerkin method," Journal of Applied Mathematics and Bioinformatics, vol. 1, pp. 343-359, 2011.

[10] O. R. N. Samadi and E. Tohidi, "The spectral method for solving system of integral equations," Journal of Applied Mathematics and Computing, vol. 40, no. 1-2, pp. 477-497, 2012.

[11] T. Tang, X. Xu, and J. Cheng, "On spectral methods for Volterra integral equations and the convergence analysis," Journal of Computational Mathematics, vol. 26, no. 6, pp. 825-837, 2008.

[12] E. Tohidi and O. R. N. Samadi, "Optimal control of nonlinear Volterra integral equations via Legendre polynomials," IMA Journal of Mathematical Control and Information, vol. 30, no. 1, pp. 67-83, 2013.

[13] Z. Wan, Y. Chen, and Y. Huang, "Legendre spectral Galerkin method for second-kind Volterra integral equations," Frontiers of Mathematics in China, vol. 4, no. 1, pp. 181-193, 2009.

[14] C. Canuto, M. Y. Hussaini, A. Quarteroni, and T. A. Zang, Spectral Methods: Fundamentals in Single Domains, Springer, 2006.

[15] C. K. Qu and R. Wong, "Szegö's conjecture on Lebesgue constants for LEGendre series," Pacific Journal of Mathematics, vol. 135, no. 1, pp. 157-188, 1988.

[16] J. Hofbauer and K. Sigmund, The Theory of Evolution and Dynamical Systems, vol. 7 of London Mathematical Society Student Texts, Cambridge University Press, London, UK, 1988.

[17] S. Olek, "An accurate solution to the multispecies Lotka-Volterra equations," SIAM Review, vol. 36, no. 3, pp. 480-488, 1994.

[18] F. Brauer and C. Castillo-Chavez, Mathematical Models in Population Biology and Epidemiology, Springer, 2000.

[19] D. Cooke and R. W. Hiorns, The Mathematical Theory of the Dynamics of Biological Populations II, Academic Press, 1981.

[20] J.-H. He, "Homotopy perturbation method for solving boundary value problems," Physics Letters A, vol. 350, no. 1-2, pp. 87$88,2006$.

[21] A. J. Lotka, Elements of Physical Biology, Williams and Wilkins, Baltimore, Md, USA, 1925.

[22] V. Volterra, "Variations and fluctuations of the number of individuals in animal species living together," in Animal Ecology, NewYork, NY,USA, McGraw-Hill, 1931.

[23] J. D. Murray, Mathematical Biology, Springer, Berlin, Germany, 1993.

[24] Ş. Yüzbaşı, "Bessel collocation approach for solving continuous population models for single and interacting species," Applied Mathematical Modelling, vol. 36, no. 8, pp. 3787-3802, 2012.

[25] H. Adibi and A. M. Rismani, "On using a modified Legendrespectral method for solving singular IVPs of LANe-Emden type," Computers \& Mathematics with Applications, vol. 60, no. 7, pp. 2126-2130, 2010.

[26] A. M. Rismani and H. Monfared, "Numerical solution of singular IVPs of Lane-Emden type using a modified LEGendrespectral method," Applied Mathematical Modelling, vol. 36, no. 10, pp. 4830-4836, 2012. 
[27] S. Pamuk and N. Pamuk, "He's homotopy perturbation method for continuous population models for single and interacting species," Computers \& Mathematics with Applications, vol. 59, no. 2, pp. 612-621, 2010.

[28] B. Batiha, M. S. M. Noorani, and I. Hashim, "Variational iteration method for solving multispecies Lotka-Volterra equations," Computers \& Mathematics with Applications, vol. 54, no. 7-8, pp. 903-909, 2007.

[29] S. Pamuk, "The decomposition method for continuous population models for single and interacting species," Applied Mathematics and Computation, vol. 163, no. 1, pp. 79-88, 2005.

[30] E. Yusufoğlu and B. Erbaş, "He's variational iteration method applied to the solution of the prey and predator problem with variable coefficients," Physics Letters A, vol. 372, no. 21, pp. 38293835, 2008. 


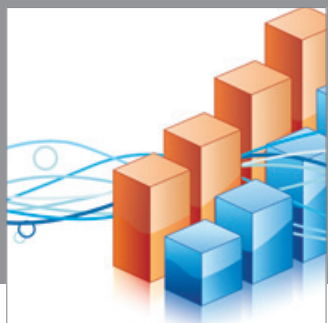

Advances in

Operations Research

mansans



The Scientific World Journal
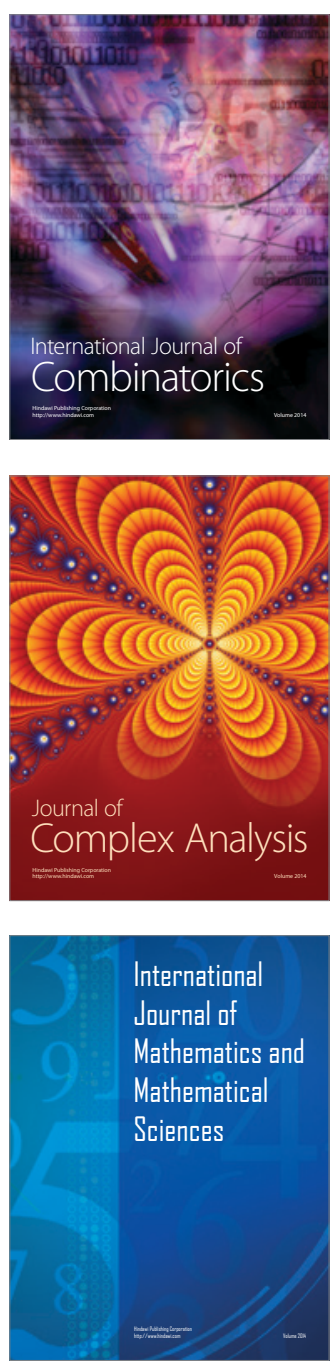
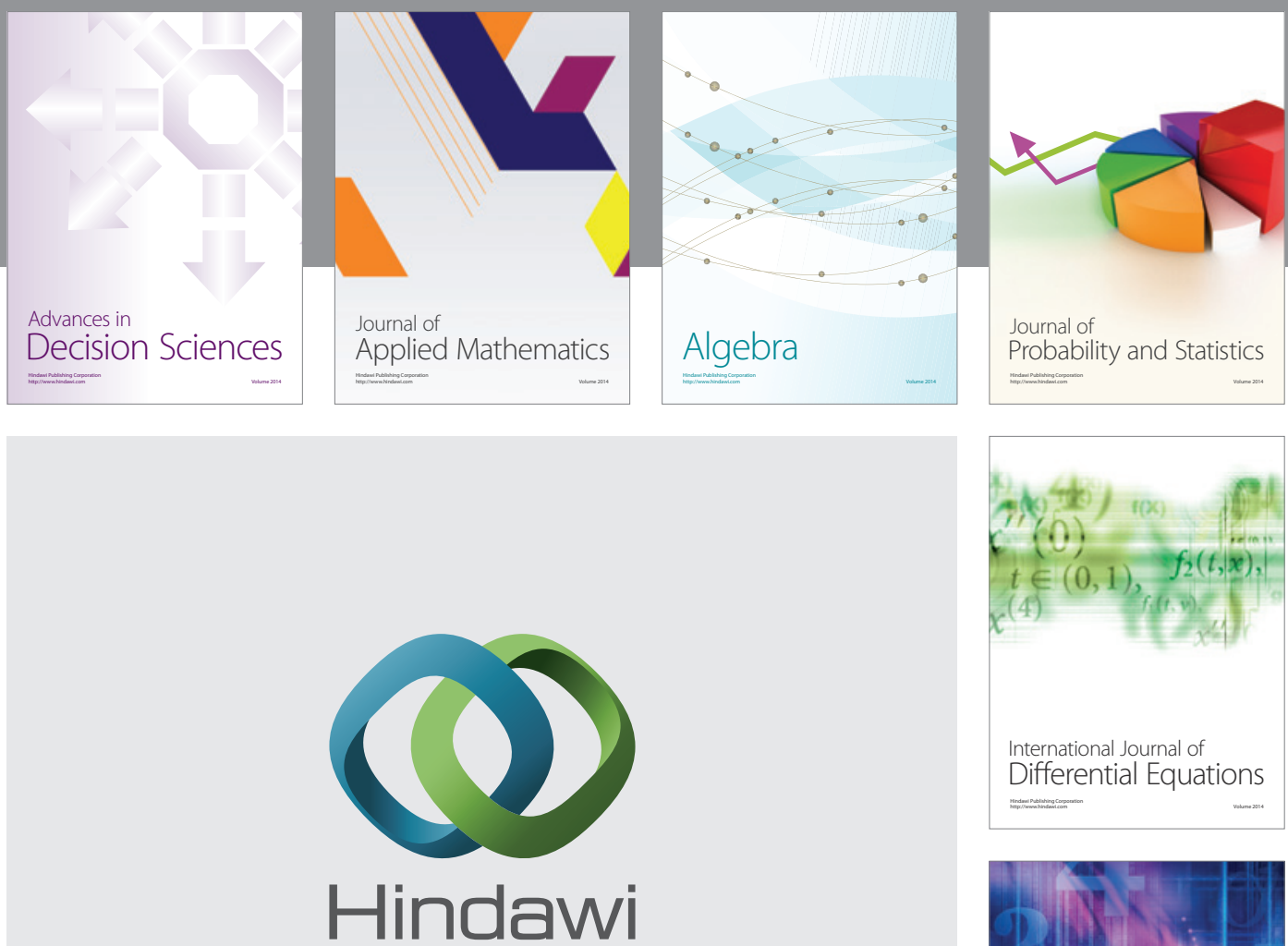

Submit your manuscripts at http://www.hindawi.com


Journal of

Function Spaces

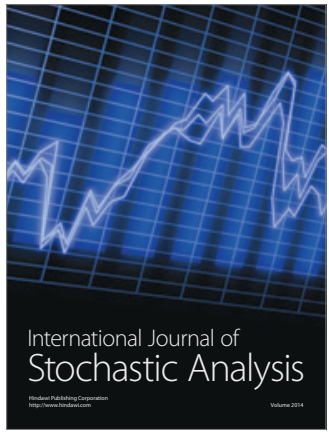


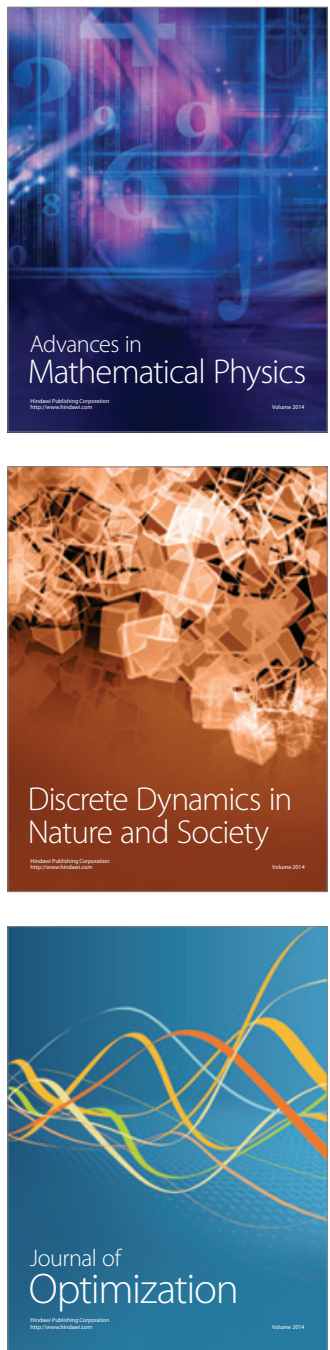